\title{
Comment développer ses compétences en TIC? L'expérience des personnes expertes de divers milieux du Réseau CompéTICA
}

Auteurs

Jeanne Godin, professeure, Université de Moncton, Canada, jeanne.godin@umoncton.ca

Viktor Freiman, professeur, Université de Moncton, Canada, viktor.freiman@umoncton.ca 


\section{REVUE HYBRIDE DE L'ÉDUCATION}

\section{Résumé}

Cet article identifie les attitudes, les défis et la formation qui ont permis à 42 personnes expertes du Canada atlantique et partenaires du Réseau CompéTICA d'acquérir des compétences en TIC. Les personnes expertes proviennent des milieux de l'éducation, de l'enseignement postsecondaire, des entreprises privées, du gouvernement et d'organismes à but lucratif, sans but lucratif et communautaires. À travers une méthode Delphi, il est démontré que la formation informelle est un facteur important dans la maitrise des compétences en TIC et que des attitudes spécifiques peuvent aider à les acquérir. À la suite des résultats obtenus, un cadre conceptuel est présenté.

Mots clés : compétences numériques; attitudes; littératie numérique; éducation; approche écosystémique 


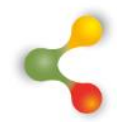

\section{REVUE HYBRIDE DE L'ÉDUCATION}

\section{Problématique}

Les origines du Réseau des partenaires CompéTICA ${ }^{1}$, dont le travail fait l'objet du présent article, remontent aux débuts des années 2000 lorsque l'enjeu du développement des compétences en technologie de l'information et de la communication (TIC) a été mis en évidence dans divers milieux éducatifs au Nouveau-Brunswick, en Atlantique et ailleurs. C'est à ce moment qu'a été fondée une organisation à but non lucratif, I'Association pour l'avancement pédagogique des TIC en Atlantique $(\mathrm{APTICA})^{2}$, qui menait, lors de ses congrès annuels, des échanges de pratiques innovatrices. En offrant des ateliers de développement professionnel à des centaines de personnes enseignantes du milieu scolaire et postsecondaire, ces congrès visaient l'intégration pédagogique d'outils numériques pouvant soutenir le processus d'enseignementapprentissage (Freiman et al., 2015).

En 2010, alors que I'APTICA surfait sur la popularité de ses congrès et d'autres projets de collaboration à l'échelle atlantique, nationale et internationale, il est devenu évident que ces succès n'avaient pas mené à un usage inclusif et équitable des technologies numériques. Pourtant, ce résultat d'apprentissage transdisciplinaire est toujours prescrit depuis 2000, comme indiqué dans le référentiel du cadre théorique commun à tous les programmes d'études de la maternelle à la $12^{\mathrm{e}}$ année au Nouveau-Brunswick francophone. II semble que l'habileté à "utiliser judicieusement les technologies de l'information et de la communication (TIC) dans des situations variées " (Gouvernement du NouveauBrunswick, 2003, Gouvernement du Nouveau-Brunswick, 2018, p. 8), bien que réclamée, ne soit pas atteinte (Chiasson 2019; Léger et Freiman, 2016).

En effet, malgré plusieurs initiatives gouvernementales visant l'intégration des TIC dans les écoles pendant les années 2000, celles-ci n'ont pas dépassé le stade de projet pilote ou d'expérimentation ponctuelle ${ }^{3}$. Finalement, contrairement à certaines disciplines (comme en mathématiques et en sciences) dans lesquelles des examens provinciaux sont menés annuellement, aucune mesure provinciale n'a été créée pour mesurer les progrès des élèves en matière de compétences en TIC durant leur parcours scolaire. II est donc devenu important de se questionner sur ces constats, ainsi qu'au-delà du système scolaire, soit dans d'autres contextes de vie - études - carrière.

Ainsi, en 2012-2013, les auteurs de cet article, avec d'autres membres de l'APTICA, ont entrepris des démarches de réflexion et de recherche afin d'identifier de nouvelles façons de promouvoir l'utilisation pédagogique des TIC de manière systémique, durable et efficace. Par

\footnotetext{
${ }^{1}$ Réseau de compétences en TIC en Atlantique, https://competi.ca/

2 http ://www.aptica.ca/

3 À titre d'exemples, le projet Accès direct à l'ordinateur portable (Blain, 2007) et les projets de Fonds d'Innovation en Apprentissage, tel que RoboMaTIC (Savard et Freiman, 2012).
} 


\section{6}

\section{REVUE HYBRIDE DE L'ÉDUCATION}

exemple, une table ronde a été organisée lors du Congrès de l'APTICA en 2013 avec les partenaires d'affaires et des milieux scolaire, universitaire, collégial pour identifier les enjeux des compétences numériques et les besoins d'un travail plus concerté, à partir de partenariats intersectoriels et multidisciplinaires dans une perspective écosystémique. Entre autres, comme le Conseil de recherche en sciences humaines (CRSH) du Canada, nous cherchions à identifier comment développer chez toutes les personnes canadiennes les habiletés «de trouver, d'organiser, de comprendre, d'évaluer et de créer de l'information au moyen de la technologie numérique pour pouvoir fonctionner dans leur milieu de travail et dans la vie de tous les jours » $(\mathrm{CRSH}, 2010$, p. 1). De plus, cette même année, les résultats d'une étude internationale de l'Organisation de coopération et de développement économiques (OCDE) portant sur l'Évaluation des compétences des adultes (PEICA), ont été publiés démontrant des lacunes chez plusieurs adultes canadiens âgés de 16 à 65 ans au niveau des habiletés en résolution de problèmes dans les environnements riches en technologies (RP-ERT), une compétence qui touche explicitement le numérique (OCDE, 2013).

Une analyse plus détaillée montre d'autres défis dont la présence bien articulée d'une "fracture numérique ». Par exemple, bien que sans surprise, les jeunes et les adultes canadiens âgés de 16 à 24 ans et de 25 à 34 ans sont les plus compétents en matière de compétences RP-ERT, il reste tout de même que $9 \%$ des plus jeunes obtiennent un score inférieur au niveau 1 (ce qui veut dire qu'ils n'ont pas d'accès aux technologies numériques même en ayant fait des études universitaires). De plus, seulement un très petit nombre d'adultes atteignent le niveau 3 (le plus élevé de cette habileté), ce qui semble indiquer les frontières possibles d'un nouveau type de fracture numérique tant au niveau national que provincial ${ }^{4}$.

En outre, ces préoccupations ont nourri des initiatives fédérales sur le plan de l'économie numérique impliquant plusieurs organismes, dont le $\mathrm{CRSH}$ du Canada qui a organisé des synthèses de connaissances et fait de l'économie numérique un domaine prioritaire. C'est donc en réponse à ces constats, avec le soutien du programme de Développement de partenariat que le Réseau CompéTICA ${ }^{5}$ a été fondé en 2014 dans le but de développer un partenariat stratégique multisectoriel et multidisciplinaire pour comprendre l'écosystème, l'adaptabilité et le transfert de compétences numériques. Le Réseau comprend les domaines de l'éducation de la maternelle à la $12^{\mathrm{e}}$ année, de l'enseignement postsecondaire, des entreprises, du gouvernement provincial, des organisations à but lucratif et sans but lucratif et des communautés (Freiman et al., 2016).

Afin de répondre aux questions suivantes 1) Comment développet-on ces habiletés ? 2) Quels rôles y jouent les attitudes envers le numérique? 3) Quels sont les défis pour devenir des personnes

\footnotetext{
${ }^{4}$ Les écarts sont encore plus grands dans certaines provinces, dont le Nouveau-Brunswick.

${ }^{5}$ https://CompéTI.CA/
} 


\section{$\&$}

\section{REVUE HYBRIDE DE L'ÉDUCATION}

compétentes en matière de TIC ?, le Réseau CompéTICA a entrepris la présente étude Delphi.

\section{Revue de littérature}

À l'instar de résultats PEICA de l'OCDE (2013), la synthèse des connaissances portant sur le développement des compétences en littératie numérique pour la future main-d'œuvre canadienne effectuée par Martinovic et Freiman (2013) a montré que les personnes ayant des niveaux plus élevés de compétences numériques semblent être mieux outillées pour s'intégrer au marché d'emploi. Les auteurs citent en exemple Washbon (2012) qui soutient que la technologie transforme les pratiques professionnelles de base dans la mesure où les compétences requises par le marché du travail deviennent similaires à celles acquises dans les programmes postsecondaires, même dans les emplois pour lesquels un diplôme d'études secondaires aurait été suffisant. D'autres synthèses des connaissances plus récentes ont confirmé qu'une compréhension plus profonde de comment les compétences numériques sont acquises (Freiman et al., 2018; Powell, 2017) semble être encore un enjeu de taille en recherche et en pratique.

En plus de ces synthèses des connaissances sur des thématiques spécifiques, notre équipe a réalisé deux tours de recension des écrits sur la question de l'apprentissage des compétences numériques; cette recension est constamment mise à jour (Godin et al., 2020). Entre autres, nos analyses démontrent que la plupart des recherches se font dans des environnements spécifiques et en silos. Par exemple, Raish et Rimland (2016) ont étudié la manière dont les employeurs percevaient les compétences critiques en littératie informationnelle, Černochová, Voňková, Štípek et Černá (2018) ont mesuré la façon dont les personnes apprenantes évaluent leurs propres compétences numériques et Phuapan, Viriyavejakul et Pimdee (2016) ont cherché à déterminer quelles compétences en littératie numérique étaient les plus importantes pour la clientèle étudiante universitaire. Siddiq, Gochyyev et Wilson (2017) ont quant à eux validé le test Learning in Digital Networks - ICT Literacy (LDN$I C T$ ) et analysé comment l'apprentissage est réalisé dans les réseaux numériques en milieu scolaire. Greene (2018) souligne également l'absence de la connaissance liée à la littératie numérique dans un contexte d'enseignement et d'apprentissage du point de vue de la personne enseignante. Pour leur part, Oberlander, Beinicke et Bipp (2020) ont combiné une recension des écrits sur les compétences numériques avec des entrevues en lien avec des évènements critiques du milieu de travail. Toutes les recherches mentionnées ci-dessus se sont concentrées sur des populations spécifiques dans des domaines spécifiques tels que les écoles, les universités et le milieu de travail.

Bien qu'étroitement liée à notre recherche, l'adaptation du cadre d'apprentissage numérique de l'UNESCO pour la pratique enseignante en Irlande (Butler et al., 2018) ne reflète pas la perspective canadienne. Toutefois, le rapport récent de Ehlers et Kellermann (2019) est semblable 


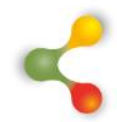

\section{REVUE HYBRIDE DE L'ÉDUCATION}

à notre recherche dans leur approche holistique et systémique avec des personnes expertes, mais vise le domaine de l'enseignement supérieur et est axé sur les " compétences futures ". Grâce à une méthode Delphi, ces derniers définissent ce terme comme la "capacité à agir avec succès sur un problème complexe dans un contexte d'action futur inconnu» [traduction libre] (p. 3). Selon eux, une personne compétente peut agir de manière auto-organisée et sa performance doit être visible. Sachant que de nombreuses études se sont limitées aux compétences numériques, Ehlers et Kellermann se concentrent sur les compétences futures visant un champ plus large. Leurs recommandations plaident en faveur d'une intégration plus forte des ressources numériques dans l'infrastructure, l'enseignement et l'apprentissage en milieu universitaire. Cependant, ils soulignent le besoin que le personnel académique soit formé et doté à la fois des compétences et des connaissances nécessaires.

Cette revue de la littérature ne nous a pas permis de découvrir les recherches qui ont été menées pour identifier les attitudes, les défis et les stratégies des personnes expertes de divers contextes, soit de regarder simultanément dans une perspective écosystémique plus globale à travers les contextes d'apprentissage et la durée de vie. C'est donc pour pallier ces lacunes que le Réseau CompéTICA a entrepris la présente recherche.

\section{Cadre conceptuel}

L'utilisation d'approches théoriques holistiques et systémiques, qui mettent l'accent sur les liens entre les différentes composantes de l'écosystème numérique au Canada atlantique, était un choix plausible pour établir un partenariat et développer le Réseau CompéTICA avec un programme de recherche collaboratif. À travers une approche théorique ancrée (Paillé, 1994), les compétences numériques sont étudiées d'un point de vue épistémologique plus inductif, aligné sur celui de Gapski (2007). Comme suggéré par Calvani, Ranieri et Fini (2009), le cadre conceptuel intègre trois dimensions: la dimension technologique (la capacité d'explorer de manière flexible de nouveaux contextes technologiques), la dimension cognitive (la capacité de lire, choisir, interpréter et évaluer l'information en ce qui concerne sa pertinence et sa validité) et la dimension éthique (la capacité d'interagir avec les autres de manière constructive et responsable).

Ce type de recherche est approprié pour créer une culture durable de partenariat efficace avec un impact socioéconomique significatif (Orunsolu et al., 2010). Comme préconisé par Inns, Nunn et Surendranath (2012), l'approche collaborative a permis de réunir des partenaires en quête d'une meilleure connectivité, issus de plusieurs milieux, soit de l'éducation, de l'enseignement, de la formation postsecondaires, de l'alphabétisation et des organisations communautaires sur le développement des compétences numériques, des organisations à but non lucratif, des agences gouvernementales et du secteur privé. 


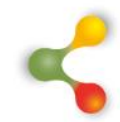

\section{REVUE HYBRIDE DE L'ÉDUCATION}

Quoiqu'en 2021, plusieurs nouveaux cadres conceptuels en compétences numériques soient disponibles, comme le Cadre de référence de la compétence numérique (Gouvernement du Québec, 2019), le Réseau CompéTICA opta, dès 2014, pour le modèle de littératie numérique d'HabiloMédias ${ }^{6}$, qui représentait à notre avis, le modèle le plus holistique et écosystémique, en plus d'être ancré dans le contexte canadien. Ce modèle, basé sur le cadre DigEuLit (European Framework for Digital Literacy) (Martin, 2005) et sur le modèle de Jenkins et al. (2006), définit le développement de la littératie numérique à partir de trois catégories de compétences, soit l'utilisation, la compréhension et la création. Comme le montre la figure 1, il demeure important de promouvoir d'abord l'accès aux technologies pour toutes et tous. II fait également référence à la fracture numérique (ligne brisée du modèle) toujours présente dans certains milieux (OCDE, 2019 ; Zorn, 2020) où il existe des lacunes liées à la connectivité (incluant l'accès Internet) où, par conséquent, de nombreuses personnes ne sont pas en mesure d'utiliser la technologie.

\footnotetext{
6 Le centre canadien d'éducation aux médias et de littératie numérique, https://habilomedias.ca/ 


\section{8}

\section{REVUE HYBRIDE DE L'ÉDUCATION}

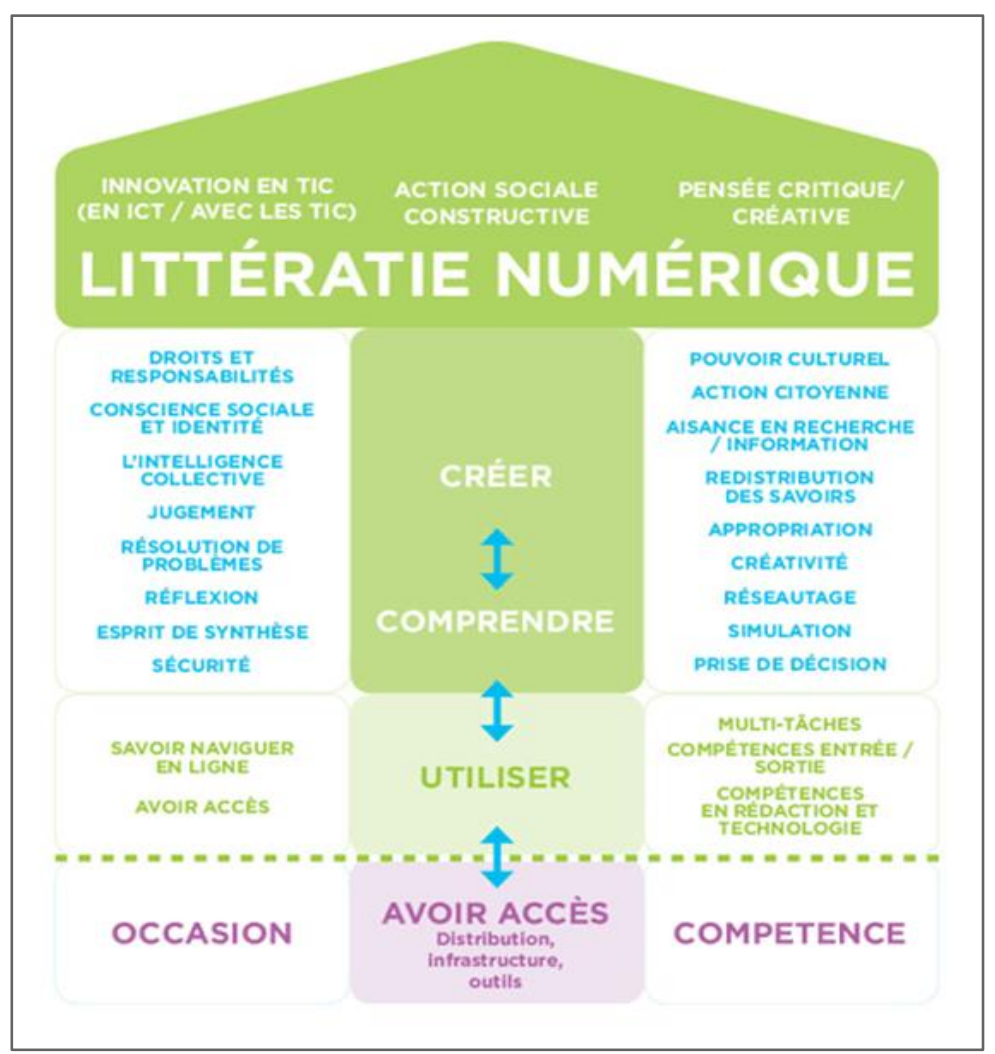

Figure 1 : Cadre de référence HabiloMédias

Toutefois, comme le montre le modèle, l'accès ne fournit pas automatiquement des occasions d'usage compétent et responsable. Le chemin vers des niveaux plus élevés de littératie numérique pourrait être différent pour chaque citoyenne et citoyen aux prises avec des défis numériques. Les résultats préliminaires de quelques études menées au sein de CompéTICA (LeBlanc et al., 2015) rapportent que les compétences techniques dominent les pratiques pédagogiques, tandis que les compétences non techniques sont non seulement obscures et sousdéveloppées, mais également difficiles à évaluer (Freiman et al., 2016 ; Freiman et al., 2017). Par conséquent, une recherche visant à approfondir les compétences et les attitudes et identifier les défis soulevés par les personnes expertes fut entreprise afin d'obtenir une perspective plus claire de leur parcours d'apprentissage et de permettre d'inspirer les pratiques éducatives futures.

Dans le contexte de cette recherche, la formation formelle est définie comme un environnement structuré et organisé (université, école ou collège) menant à des diplômes ou des certifications, tandis que la formation informelle réfère à des expériences d'apprentissage en dehors 


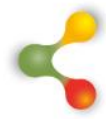

\section{REVUE HYBRIDE DE L'ÉDUCATION}

d'un environnement d'apprentissage formel traditionnel comme congrès, sessions de travail, expériences quotidiennes, autoformatrices, individuelles ou non, sur les lieux de travail ou non (Cristol et Muller, 2013). Eagly et Chaiken (1993) définissent l'attitude comme "une tendance psychologique qui s'exprime en évaluant une entité particulière avec un certain degré de faveur ou non » [traduction libre] (p. 1). Dans la théorie du comportement planifié (Ajzen 1991), les attitudes affectent notre intention d'effectuer un certain comportement. Elles sont donc importantes dans le développement des compétences numériques. En fait, depuis 2008, Venkatesh et Bala soutiennent l'importance de l'attitude dans leur modèle d'acceptation des nouvelles technologies et Hamutoglu, Savasci et SezenGultekin (2019) démontrent leur pertinence dans l'acquisition de compétences numériques.

\section{Méthodologie}

Face aux défis de l'ambiguïté en matière d'acquisition de compétences en TIC ainsi qu'aux caractéristiques du Réseau CompéTICA, cette étude, grâce à la méthode Delphi, vise à identifier les attitudes, les défis et la formation qui ont permis à des personnes expertes du Canada atlantique d'acquérir des compétences en TIC. Comme le suggèrent ses racines conceptuelles, la méthode Delphi permet de trouver des réponses dans des données provenant de sources multiples (Baker et al., 2006). Le Réseau CompéTICA a effectué, auprès de 42 personnes expertes identifiées par les partenaires, des entrevues semi-structurées. Les discours communs qui émergent de ces personnes expertes peuvent fournir des orientations et des exemples pratiques permettant au personnel éducateur de naviguer avec succès dans la complexité des espaces et des outils numériques.

La sélection des personnes expertes est fondamentale pour la validité et la fiabilité des résultats de l'étude Delphi (Donohoe et Neddham, 2009). Baker, Lovell et Harris (2006) définissent l'expertise par les connaissances et l'étendue de l'expérience liées à une qualification professionnelle et la durée d'implication par rapport au sujet en question. Comme suggéré par ces spécialistes de la recherche, les personnes expertes ont été sélectionnées sur la base de leurs connaissances et de l'expérience acquise du sujet. Les critères d'inclusion étaient les suivants : posséder une expérience pertinente en TIC, être reconnu dans son domaine de travail, être recommandé par une personne gestionnaire ou des pairs comme étant une personne experte, être connu dans la communauté des TIC comme étant une personne experte, être un défenseur informé du sujet et être disposé à participer à la recherche. Chaque organisation partenaire impliquée dans le Réseau CompéTICA devait être représentée et par conséquent, devait recommander leurs personnes expertes.

Lors du premier tour de l'étude Delphi (2014-2015), le guide d'entrevue semi-structurée a été créé par l'équipe de recherche et validé à la fois par les références aux écrits scientifiques et par les membres du 


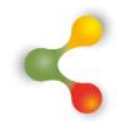

\section{REVUE HYBRIDE DE L'ÉDUCATION}

Réseau. Ainsi, une première ébauche a ensuite été envoyée par courriel à tous les partenaires du Réseau CompéTICA pour recueillir leurs commentaires et recommandations. Après ces consultations, une dernière série de questions a été élaborée. Dans le cadre du processus de validation, le guide d'entrevue a été testé avec deux personnes expertes n'ayant pas participé à l'étude afin de vérifier la clarté et la compréhension avant d'être utilisé avec les personnes participantes. Le guide d'entrevue débutait en leur demandant de définir l'expression "compétences numériques » puis d'identifier les compétences importantes. Par la suite, le guide cherchait à connaitre les attitudes nécessaires à leur acquisition suivie des défis qu'elles ont eu à surmonter. II était aussi demandé comment elles avaient atteint leur niveau de compétences. La seconde partie du questionnaire visait à comprendre comment les compétences numériques étaient développées dans leur milieu de travail spécifique et se terminait en leur demandant comment elles pouvaient contribuer au Réseau CompéTICA.

À la suite de l'approbation du comité d'éthique de l'Université de Moncton, chaque partenaire a été contacté à plusieurs reprises, soit en personne lors des rencontres bi annuelles du Réseau CompéTICA, par courriel ou par téléphone afin d'identifier les personnes expertes en TIC dans leurs domaines respectifs. Dans l'ensemble, conformément aux critères susmentionnés, 42 personnes expertes furent interrogées : huit en provenance du secteur privé, huit du système scolaire $M-12$, huit de formation continue pour adultes, neuf de milieux universitaires et de collèges communautaires, quatre d'organisations d'intervention familiale et cinq d'organisations communautaires. Les entrevues ont varié de 20 à 45 minutes et ont donné lieu à plus de 900 minutes de données d'entrevue.

Les premières entrevues semi-structurées ont été menées en 2014-2015 et les premiers résultats axés sur l'éducation postsecondaire ont été rapportés dans LeBlanc et al. (2015). Les entrevues se sont poursuivies en 2016-2017. À chaque tour d'entrevues, les résultats faisaient l'objet d'une réflexion critique lors de réunions avec nos partenaires qui ont suggéré, en 2016, de commencer, en parallèle avec les entrevues, des études de cas de pratiques exemplaires pour pouvoir croiser les données d'entrevues avec les pratiques du terrain. En tout, les 42 entrevues ont été transcrites et analysées. Chaque transcription d'entrevue semi-structurée a été lue attentivement par les auteurs de cet article afin d'identifier des thèmes décrivant le mieux des attitudes, la formation reçue ou son absence et les défis spécifiques à surmonter pour devenir des personnes compétentes en TIC.

En parallèle, trois grilles étaient construites afin de dégager des catégories thématiques émergentes (Miles et Huberman, 1994). La première grille décrivait comment chaque personne experte avait appris à devenir compétente, la deuxième récoltait les attitudes nommées ainsi que leur fréquence en précisant quelles personnes expertes l'avaient exprimé tout en servant aussi à recueillir les défis rencontrés. Enfin, une troisième 


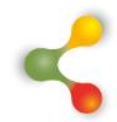

\section{REVUE HYBRIDE DE L'ÉDUCATION}

grille servait à récolter des verbatims pertinents qui explicitaient leurs propos. Les résultats ont été discutés lors d'une réunion de partenariat afin d'obtenir un consensus éclairé concernant l'interprétation de ceux-ci. À l'étape finale de l'étude, le modèle HabiloMédias a été ajusté et une nouvelle phase de Réseau a été conçue par les partenaires sous le nom Vision 2025. Nous présentant les résultats de ce travail dans la prochaine section. Notons que conformément aux règles d'éthique, les noms des 42 personnes expertes ont été remplacés par une lettre afin d'assurer l'anonymat : par conséquent, les 26 premières entrevues ont été identifiées par les lettres $A$ à $Z$ et les 16 suivants par la lettre $A$ suivie d'un nombre tel que $\mathrm{A} 1$ à $\mathrm{A} 16$.

Les résultats présentés dans la section suivante ont été rassemblés autour des questions suivantes: 1) Comment êtes-vous devenu une personne experte ? Quand et comment avez-vous appris ? (de manière formelle ou informelle) ; 2) Quel genre d'attitudes une personne devrait-elle avoir pour développer ses compétences numériques ?; 3) Quels sont les défis liés au développement des compétences numériques et comment avez-vous fait pour maitriser l'utilisation de la technologie?

\section{Résultats}

\section{L'ampleur de la formation informelle}

Par rapport aux modalités de formation reçue permettant de développer les compétences numériques, parmi les 41 personnes interrogées qui ont spécifiquement répondu à la question, moins de $20 \%$ affirment avoir suivi une formation formelle en littératie numérique comparé à près de la moitié qui ne semblent pas en avoir reçu. Le dernier tiers a déclaré avoir appris d'une combinaison de formations formelles et informelles. Sur les 21 personnes expertes ayant une formation formelle, une seule a suivi à la fois un cours au secondaire et à l'université. Deux des personnes expertes n'ont pas précisé quel type de formation formelle elles avaient reçue. Les autres personnes expertes ont reçu une formation formelle dans le cadre d'un cours à l'école, au collège ou à l'université. Les huit autres personnes expertes décrivent leur formation formelle comme un cours en ligne ou une formation offerte sur le lieu de travail. Lors de la description du contexte informel d'acquisition de compétences numériques, de nombreuses manières ont été mentionnées, telles que : «essais et erreurs », être « autodidacte » ou apprendre «sur le tas ».

"Je n'ai jamais eu de cours d'informatique. C'est beaucoup plus essais-erreurs, la curiosité et les amis qui m'ont aidé. » (Personne A5).

«Par curiosité parce que j'aimais ça, parce que je voulais apprendre, j'avais des personnes autour de moi qui m'encourageaient à apprendre. Je n'ai jamais eu peur de fouiller, mais j'aime la machine, j'aime la 


\section{$\&$}

\section{REVUE HYBRIDE DE L'ÉDUCATION}

technologie donc je voulais en savoir plus » (Personne D).

«Quand je dis essai-erreur, c'est de voir l'outil, de trouver de la documentation, de voir ce que les autres ont fait, de l'essayer, adapter les choses, développer soimême les compétences que j'ai besoin en regardant ce qui se fait ailleurs, donc sur des réseaux, avec mon réseau personnel d'apprentissage, et des trucs comme ça » (Personne M).

«Puis depuis, je dirais depuis un an ou deux on n'a pu de formation continue vraiment là. On apprend sur le tas... on apprend à force de pratiquer... Ma secrétaire aussi elle apprend à force de pratiquer » (Personne $\mathrm{P}$ ).

Elles ont également décrit la formation informelle comme la réception de l'aide de leurs pairs (Personne $Z$ ), de collègues (Personne A6) ou de membres de la famille (Personne A14). Comme le conclue Personne A11, la formation formelle se résume par «fouille, fouille, fouille».

Quant aux personnes expertes qui ont bénéficié de formations formelles et informelles, les Personnes $\mathrm{V}$ et $\mathrm{A} 10$ ont expliqué que, dans leur contexte de travail, ils adhèrent au principe 70-20-10, "où vous apprenez $70 \%$ de vos collègues de travail, $20 \%$ est transactionnel où vous obtenez une formation à vos frais et à votre rythme et $10 \%$ est de la formation formelle est fournie par votre employeur » (Personne V).

\section{Les attitudes à privilégier}

Les personnes expertes ont précisé les attitudes qui favorisent l'apprentissage des TIC et le développement des compétences numériques. Le tableau 1 montre que l'attitude la plus souvent mentionnée (par 17 personnes expertes) est l'ouverture d'esprit, soit d'être ouvert à l'apprentissage de compétences numériques. Elles ont également indiqué que la patience, la pensée positive et la curiosité sont très importantes. Personne $\mathrm{H}$ résume bien ceci : "Une attitude positive, une ouverture d'esprit et je pense aussi qu'il ne faut pas se laisser influencer par les de mauvaises expériences du passé ».

Plusieurs attestent également qu'être preneur de risque, débrouillard et autodidacte sont des attitudes essentielles. De son côté, Personne A12 est d'avis que la persévérance doit être au rendez-vous surtout lorsqu'on procède par essaie-erreur et ajoute qu'il ne faut pas craindre l'échec, mais y faire face avec courage. De son côté, Personne M soutient qu'il faut parfois remettre en question les idées préconçues "D'une part, il y a des personnes qui arrivent avec des préconçues (sic) de comment les outils informatiques fonctionnent, donc c'est un peu de les désensibiliser à ce qu'ils ont fait ». Sur ce sujet, Personne $C$ ajoute qu'il ne faut pas forcer les gens: "Je ne veux pas dire de forcer, mais c'est de trouver et faire en sorte que les gens parlent entre eux autres pour dire : on 


\section{$\&$}

\section{REVUE HYBRIDE DE L'ÉDUCATION}

croit beaucoup dans ces compétences-là et on va y mettre le temps. Si on croit que la musique est importante dans notre école on va y accorder du temps... de l'importance. »

Plusieurs autres thèmes ont été ressortis concernant les attitudes permettant de devenir des personnes compétentes en TIC, soit le vouloir apprendre (Personne A9), la volonté d'y mettre des efforts (Personne O) et enfin tel que décrit par Personne A11, il faut avancer avec courage : «II faut être innovateur, dans le sens d'avoir le courage d'essayer de nouvelles choses parce que souvent on est régressif. »

Le besoin ou la nécessité (sentiment d'urgence) d'apprendre (Personne A12) serait une source additionnelle de motivation qui mène à l'apprentissage. De son côté, Personne A5 a soulevé l'importance de se connaitre afin d'être en mesure d'identifier ce que l'on sait ou maitrise versus à ce qu'il nous reste à apprendre. La résilience (Personne A8) et le dépassement (Personne A11) peuvent permettre la mise en place d'initiatives signifiantes qui permettent d'apprivoiser les nouvelles technologies. Pour sa part, Personne $\mathrm{N}$ a soulevé qu'apprendre de nouvelles techniques ou compétences par le jeu a fait partie de son apprentissage tout en précisant que prétendre de jouer avec un logiciel lui a permis de découvrir des trucs et de partager avec ses collègues.

Tableau 1 : Fréquence et variété des attitudes proposées par les personnes expertes

\section{Attitudes}

\begin{tabular}{|c|c|c|c|c|c|}
\hline Les plus souvent & 3-4 experts & 2 experts & \multicolumn{3}{|c|}{ Mentionné par 1 expert } \\
\hline $\begin{array}{l}\text { Ouverture } \\
17\end{array}$ & $\begin{array}{l}\text { Prise de } \\
\text { risques } 4\end{array}$ & $\begin{array}{l}\text { Pas peur des } \\
\text { nouveautés }\end{array}$ & $\begin{array}{l}\text { On joue on } \\
\text { partage }\end{array}$ & Flexibilité & $\begin{array}{l}\text { Anticiper } \\
\text { obstacles }\end{array}$ \\
\hline Patience 8 & $\begin{array}{l}\text { Autonome/ } \\
\text { autodidacte } 3\end{array}$ & Pas être forcé & Courageux & $\begin{array}{l}\text { Nécessité/ } \\
\text { besoin }\end{array}$ & $\begin{array}{l}\text { Pas peur de } \\
\text { l'échec }\end{array}$ \\
\hline Positif 7 & $\begin{array}{l}\text { Débrouillardise } \\
3\end{array}$ & Vouloir/volonté & $\begin{array}{l}\text { Avant- } \\
\text { gardiste }\end{array}$ & Polyvalence & $\begin{array}{l}\text { Se connaitre / } \\
\text { savoir ce que tu } \\
\text { connais ou pas }\end{array}$ \\
\hline \multirow[t]{5}{*}{ Curiosité 7} & & Persévérance & $\begin{array}{l}\text { Tenir à jour/ } \\
\text { informé }\end{array}$ & $\begin{array}{l}\text { Pour le } \\
\text { changement }\end{array}$ & $\begin{array}{l}\text { Accorder de } \\
\text { l'importance }\end{array}$ \\
\hline & & Collaboration & Motivation & Éviter préjugés & Logique \\
\hline & & Essaie-erreur & $\begin{array}{l}\text { Attention aux } \\
\text { détails }\end{array}$ & $\begin{array}{l}\text { Réagir aux } \\
\text { changements }\end{array}$ & Apprivoiser \\
\hline & & & Dépassement & $Y$ croire & Initiative \\
\hline & & & Résilience & Découvrir & Signifiant \\
\hline
\end{tabular}




\section{$\&$}

\section{REVUE HYBRIDE DE L'ÉDUCATION}

\section{Les défis à surmonter}

Quels sont les défis rencontrés par les personnes expertes alors qu'elles parcouraient leurs trajectoires personnelles ainsi que ceux qu'elles croient qui pourraient empêcher les autres de développer leurs compétences numériques? Les personnes expertes sont unanimes sur le fait que les deux défis majeurs sont la peur et le temps (tableau 2). La peur est décrite comme la peur du changement, la crainte de ne pas être capable de se tenir à jour face aux nombreuses innovations qui arrivent rapidement et la peur d'échouer. Le temps mine la capacité de réussir et présente un grand défi que peu de gens semblent avoir maitrisé, alors que comme l'explique Personne A3, devenir compétent prend du temps.

Je dirais qu'un des plus grands défis c'est probablement le facteur temps... parce que dans nos écoles il y a énormément de demandes, énormément de projets et énormément d'initiatives qui sont demandés aux enseignants... ça veut dire que des fois tu n'es pas nécessairement prête mentalement à entreprendre d'autres choses. (Personne A8)

Certaines personnes expertes, comme Personne $A 5$, suggèrent que l'infrastructure et les coûts sont des défis qui découragent l'apprentissage des compétences numériques :

Des fois c'est des défis financiers, donc l'institution ne peut pas nous doter d'outils. On manque de ressources financières et de matériels donc on a beau développer, on a beau vouloir cet outil-là, mais il n'est pas accessible, il est trop cher ou l'institution ne veut pas le supporter.

Le tableau suivant présente les défis les plus mentionnés par les personnes expertes ainsi que la variété d'obstacles perçus dans leur milieu respectif. 


\section{REVUE HYBRIDE DE L'ÉDUCATION}

\section{Tableau 2 : Fréquence et variété des défis}

\begin{tabular}{|c|c|c|c|c|c|}
\hline Les plus souvent & 3-4 experts & 2 experts & \multicolumn{3}{|c|}{ Mentionné par 1 expert } \\
\hline Peur (13) & $\begin{array}{l}\text { Ministère } \\
\text { programme }\end{array}$ & Manque de transfert & $\begin{array}{l}\text { Création de } \\
\text { contenus } \\
\text { perdus }\end{array}$ & $\begin{array}{l}\text { Manque } \\
\text { d'intérêt des } \\
\text { jeunes }\end{array}$ & $\begin{array}{l}\text { Ressources } \\
\text { anglophones }\end{array}$ \\
\hline Temps (10) & $\begin{array}{l}\text { Curriculum/ } \\
\text { évaluation }\end{array}$ & $\begin{array}{l}\text { Présupposition des } \\
\text { acquis }\end{array}$ & $\begin{array}{l}\text { Quand ca } \\
\text { manque: } \\
\text { Panique }\end{array}$ & $\begin{array}{l}\text { Formé à } \\
10 \% \ldots \text { pas } \\
100 \%\end{array}$ & $\begin{array}{l}\text { Points d'accès } \\
\text { saturés dans } \\
\text { écoles }\end{array}$ \\
\hline $\begin{array}{l}\text { Changements, } \\
\text { nouveautés, se } \\
\text { tenir à jour (7) }\end{array}$ & $\begin{array}{l}\text { Après embauche } \\
\text { offrir plus de } \\
\text { formations }\end{array}$ & $\begin{array}{l}\text { Chacun est laissé à } \\
\text { lui-même }\end{array}$ & $\begin{array}{l}\text { Manque } \\
\text { connaissances } \\
\text { compétences } \\
\text { pratique }\end{array}$ & $\begin{array}{l}\text { S'ajuster aux } \\
\text { changements }\end{array}$ & $\begin{array}{l}\text { Équipement de } \\
\text { dernière heure? }\end{array}$ \\
\hline \multirow{5}{*}{$\begin{array}{l}\text { Infrastructure } \\
\text { internet instables } \\
\text { (6) }\end{array}$} & Savoir lire & $\begin{array}{l}\text { Trous dans } \\
\text { l'apprentissage }\end{array}$ & $\begin{array}{l}\text { Qui demande } \\
\text { reçoit.. }\end{array}$ & $\begin{array}{l}\text { Nouveau } \neq \\
\text { mieux }\end{array}$ & $\begin{array}{l}\text { Relation de } \\
\text { travail }\end{array}$ \\
\hline & $\begin{array}{l}\text { Formation des } \\
\text { formateurs }\end{array}$ & $\begin{array}{l}\text { Se faire contrôler par } \\
\text { une machine }\end{array}$ & $\begin{array}{l}\text { Fermeture } \\
\text { (attitude) }\end{array}$ & Persévérance & $\begin{array}{l}1 \text { mentor pour } \\
38 \text { écoles }\end{array}$ \\
\hline & Coûts & Manque de matériel & Individualisé & Bons mots-clés & Pupitres \\
\hline & & Chacun est unique & Fiabilité & Sécurité & Réticences \\
\hline & & & Âge & Plein potentiel & Découragement \\
\hline
\end{tabular}

Lorsque l'on analyse plus en profondeur les réponses des personnes expertes portant plus précisément sur les défis du milieu éducatif, on peut ajouter le manque de ressources francophones comparativement à une très grande disponibilité de ressources anglophones (Personne T). On mentionne aussi les ressources créées par le passé, mais dont le format n'est plus transférable dans les nouveaux logiciels ce qui décourage même les plus motivés (Personnes A11 et A3). Ces expériences négatives rendent les personnes réticentes aux changements. Les mentors en application des technologies qui par le passé étaient plus nombreux dans les districts scolaires pour assister le personnel dans leur démarche pédagogique avec les TIC ont été extrêmement réduits, passant par exemple d'un mentor pour deux ou trois écoles à un mentor pour 38 écoles (Personne A6).

L'alphabétisation (savoir lire, Personne A14) est également soulevée. Cette personne experte explique que les personnes analphabètes ont beaucoup plus de difficultés à apprendre les compétences numériques dues aux informations sous forme écrite.

Certaines personnes expertes mentionnent également que l'évaluation des compétences numériques lors de l'embauche est inadéquate ou inexistante en précisant que, lors des entretiens d'embauche, les personnes candidates attestent être aptes en informatique alors que c'est tout le contraire une fois sur le lieu de travail, comme 


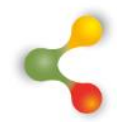

\section{REVUE HYBRIDE DE L'ÉDUCATION}

l'explique Personne A11 «ils ne sont formés qu'à $10 \%$ au lieu des $100 \%$ autoproclamés lors de l'entrevue d'embauche. On sous-estime aussi l'effort nécessaire à l'encadrement des employés... il y a des jeunes qui ont peur de dire : je ne sais pas comment... » Les mauvaises expériences passées (Personne H) avec les TIC peuvent aussi dissuader les meilleures intentions. Comme le décrivent les personnes expertes, parfois un nouveau logiciel est présenté comme plus grand et meilleur, mais ne tient pas ses promesses (Personne D). Le changement constant des technologies laisse le sentiment de ne "Jamais rien de maitrisé, jamais fini, tu deviens brulé, tu décroches » (Personne R).

\section{Discussion}

Les résultats montrent une variété considérable de trajectoires d'apprentissage pour acquérir des compétences numériques. Premièrement, les résultats suggèrent qu'une combinaison de formation formelle et informelle pourrait être un moyen pour le personnel scolaire de structurer leur enseignement, comme le soutiennent également Tinder et ses collaborateurs (2008). Meyers, Erickson et Small (2013) expliquent que les contextes d'apprentissages formel et informel ne doivent pas être décrits comme étant l'un meilleur que l'autre, mais que nous devons reconnaitre la contribution de chaque espace, qu'il soit physique ou virtuel. Ces spécialistes de la recherche ajoutent à l'expression lifelong learners (personne apprenante à vie), le terme life-wide qui pourrait être traduit par "personne apprenante dans tous les contextes de vie ». Cette perspective, beaucoup plus holistique de l'apprentissage des compétences numériques, permettrait selon cette recherche de démontrer la complexité ainsi que la nature interconnectée de ces compétences.

Deuxièmement, l'importance de favoriser la collaboration, la communication ainsi que les compétences techniques soulevées dans les résultats sont aussi présentes dans le modèle de compétences futures d'Ehlers et Kellermann (2019). Cette diversité de parcours doit être prise en compte lors du choix de pratiques pédagogiques (Akiva et al., 2016). De plus, les pratiques pédagogiques qui donnent aux personnes apprenantes l'occasion de débattre les deux côtés d'un problème apporteront de l'ouverture d'esprit ; cela a été confirmé par Léger et Freiman (2016) dans l'étude des parcours numériques des jeunes adultes 10 ans après avoir expérimenté l'utilisation d'ordinateurs portables individuels au niveau collégial.

Les attitudes mises en valeur par ces personnes expertes, soit l'ouverture d'esprit, la patience, la pensée positive et la curiosité sont certainement des attitudes qui varient au cours du cycle de vie et nécessitent certaines activités pour favoriser leur croissance. Ces qualités personnelles entrent dans une catégorie de soft skills qui semble jouer un rôle important dans le développement de l'expertise avec les TIC. Nos études de cas basées sur CompéTICA en lien avec des pratiques exemplaires confirment la valeur de ces compétences (Freiman et al., 2017). 


\section{$\&$}

\section{REVUE HYBRIDE DE L'ÉDUCATION}

La peur est un défi qui s'infiltre dans de nombreux aspects de la vie quotidienne. Comment pouvons-nous enseigner ou apprendre à surmonter la peur? Lum et Tambyah (2020) suggèrent que la pandémie de COVID19 a créé une épidémie de peur, mais que la science peut faire taire la peur. Furedi (2018) explique qu'il y a encore beaucoup de confusion sur la cause et les conséquences de la peur. II atteste que la peur a imprégné notre langage quotidien et qu'elle reflète l'esprit de notre temps. II explique qu'elle est causée par une vision fataliste de l'humanité. Par conséquent, comment une classe peut-elle nourrir l'espoir ? Comme le suggèrent Jones et Putney (2019), l'action fondée sur l'espoir peut permettre aux personnes apprenantes de maintenir l'élan dans la réalisation de leurs objectifs. L'action fondée sur l'espoir est une combinaison de croyances et d'actions.

Les résultats présentés nous ont conduits à préciser certains éléments du modèle de littératie numérique conçu par HabiloMédias (figure 2). Les changements que nous proposons au modèle sont enracinés dans le fait que le développement de la littératie numérique n'est pas statique, mais évolue plutôt avec le flux de nouveaux types de technologies au fur et à mesure de leur développement. Dans le modèle original, les lignes brisées au bas de la figure visent à montrer une fracture numérique entre les personnes «avec » et "sans " accès. Nos résultats montrent que de nombreux défis identifiés par les personnes expertes, persistent encore, même pour les personnes ayant accès. Par conséquent, notre modélisation ajoute des lignes brisées dans tout le modèle pour démontrer que la fracture numérique est présente à différents niveaux. La fracture numérique ne consiste non seulement sur le plan matériel (accès à la technologie), mais aussi sur le plan cognitif (accès aux connaissances et aux compétences), selon Calvani, Ranieri et Fini (2009). En effet, l'âge, le niveau d'éducation ou les compétences peuvent influencer la manière dont les technologies sont utilisées. Par exemple, l'analphabétisme peut empêcher une personne ayant accès à utiliser la technologie numérique de manière adéquate, ou un utilisateur peut être en mesure de créer, mais manque de compétences éthiques. 


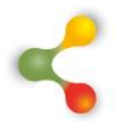

\section{REVUE HYBRIDE DE L'ÉDUCATION}

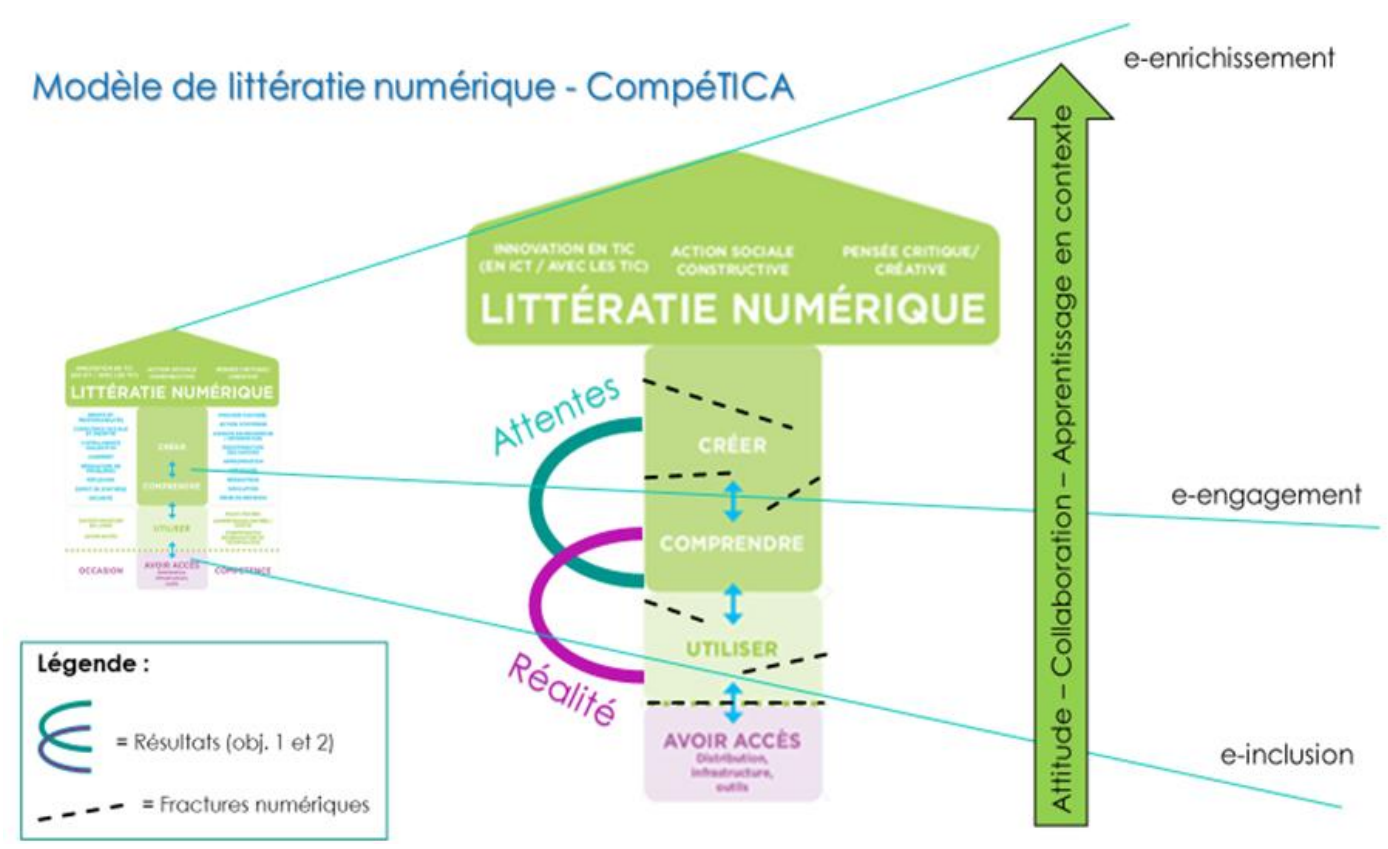

Figure 2 : Modèle de littératie numérique - CompéTICA

Les personnes expertes ont aussi signalé une disparité significative entre les attentes concernant le niveau de compétences numériques à différentes étapes de la vie par rapport au niveau de compétences que les gens possèdent en réalité à ces mêmes étapes de la vie. À droite du modèle, nous avons ajouté une flèche avec les mots Attitude, Collaboration et Apprentissage en contexte. Les attitudes spécifiques, telles que la curiosité, la persévérance et les essais et erreurs sont nécessaires pour acquérir un ensemble de compétences numériques. La collaboration et l'apprentissage en contexte ont également été identifiés comme inhérents à l'acquisition de compétences numériques. Enfin, la dernière partie du modèle représente l'aspect prospectif de notre recherche, l'avenir que nous voyons pour les compétences numériques. Nous appelons ces éléments le e-enrichissement, l'e-engagement et l'e-inclusion.

- e-inclusion : Sur le thème de l'avenir du travail, McKinsey Global Institute (2019) suggère que le retour à une croissance plus inclusive exigera, comme nous le pensons, l'énergie et l'ingéniosité combinées des chefs d'entreprise, des décideurs, des éducateurs et des secteurs sans but lucratif.

- e-enrichissement : Trouver des moyens d'aider les gens à passer du statut de simples utilisateurs de la technologie à des personnes alphabétisées numériquement. Développer de nouvelles approches pédagogiques susceptibles d'enrichir les compétences numériques de nos citoyens grâce au développement de soft skills. 


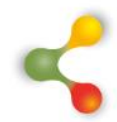

\section{REVUE HYBRIDE DE L'ÉDUCATION}

- e-engagement : eDévelopper des citoyens numériques capables de participer activement à la résolution de problèmes, d'un point de vue entrepreneurial et civique, étant donné la société numérique en constante évolution dans laquelle nous vivons.

Nous sommes aussi d'avis que certaines attitudes mises en valeur par ces personnes expertes, soit l'ouverture d'esprit, la patience, la pensée positive, la curiosité, la résilience et le dépassement varient au cours du cycle de vie et nécessitent certaines activités pour favoriser leur croissance. Ces qualités personnelles, selon nous, entrent dans une catégorie de soft skills qui semble jouer un rôle important dans le développement de l'expertise avec les TIC (Freiman et al., 2017). Ces compétences dites douces, humaines ou transversales semblent donc être essentielles à l'ère du numérique et on constate leur présence dans de nombreux curriculums (Bjørgen et Erstad, 2015). Le Nouveau-Brunswick a explicitement introduit les soft skills dans le Profil de sortie d'un élève du système scolaire acadien et francophone du Nouveau-Brunswick (Gouvernement du Nouveau-Brunswick, 2016), mais il reste à comprendre comment ils sont acquis?

La crise socioéconomique accompagnant la COVID-19 a certes amplifié la fracture numérique, mais elle l'a également mise en lumière, particulièrement pour les personnes moins nanties ou marginalisées (Zorn, 2020). Plus précisément, pour le groupe d'experts eEurope (Bouquet Jaeger, 2015), les politiques actuelles de lutte contre la fracture numérique manquent d'efficacité, car elles ne visent que sur la quantité de la présence des TIC. Selon OCDE (2019), de nombreux pays auraient intérêt à repenser l'intégration des TIC dans les programmes scolaires ainsi que la formation tout au long de la vie en luttant contre les inégalités d'accès. Plus près de nous, l'Observatoire québécois des inégalités (Zorn, 2020) soutient que le déconfinement est le moment idéal d'identifier des objectifs afin de concrétiser l'avenir que souhaite l'ensemble de la société, en ciblant les conditions de réussite pour relever les défis.

En conclusion, cet article a tenté d'informer le personnel éducatif sur les succès et les défis du développement des compétences numériques en analysant l'expérience de personnes expertes qui ont réussi à acquérir ces compétences. En identifiant les attitudes, les occasions de formation et les défis exprimés par des personnes expertes de divers domaines, nous visons à améliorer les compétences numériques sur le marché du travail et dans la vie quotidienne, contribuant ainsi au développement de citoyens numériquement compétents. Ces éléments pourraient aussi faire partie d'un outil de mesure pour évaluer ces compétences. Accroître l'utilisation significative et compétente du numérique évitera d'amplifier la fracture numérique si une formation appropriée est fournie. 


\section{REVUE HYBRIDE DE L'ÉDUCATION}

\section{Références}

Ajzen, I. (1991). The Theory of Planned Behavior. Organizational Behavior and Human Decision Processes, 50(2), 179-211. http://dx.doi.org/10.1016/0749-5978(91)90020-T

Akiva, T., Kehoe, S. et Shunn, C.D. (2016). Are we ready for citywide learning? Examining the nature of within- and between-program pathways in a community-wide learning initiative. Journal of Community Psychology, 45, 413-425

Baker, J., Lovell, K. et Harris, N. (2006). How Expert Are the Experts? An Exploration of the Concept of "Expert" within Delphi Panel Techniques. Nurse Researcher, 14, 59-70. http://dx.doi.org/10.7748/nr2006.10.14.1.59.c6010

Bjørgen, A. M. et Erstad, O. (2015). The connected child: tracing digital literacy from school to leisure. Pedagogies: An International Journal, 10(2), 113-127. http://dx.doi.org/10.1080/1554480X.2014.977290

Blain, S. (2007). Les effets de l'utilisation des ordinateurs portatifs individuels sur l'apprentissage et les pratiques d'enseignement: Rapport final. Université de Moncton. https://www.umoncton.ca/crde/files/crde/wf/wf/pdf/Projets/recherche portatif.pdf

Bouquet, B. et Jaeger, M. (2015). L'e-inclusion, un levier? Vie sociale, 3(11), 185-192. https://www.cairn.info/revue-vie-sociale-2015-3page-185.htm

Butler, D., Hallissy, M. et Hurley, J. (2018). The Digital Learning Framework: What Digital Learning can look like in Practice, An Irish Perspective. Dans E. Langran et J. Borup (dir.), Proceedings of Society for Information Technology \& Teacher Education International Conference (p. 1339-1346). Association for the Advancement of Computing in Education (AACE). https://www.learntechlib.org/primary/p/182702/

Calvani, A., Ranieri, M. et Fini, A. (2009). Assessing Students' Digital Competence in Schools. Dans T. Bastiaens, J. Dron et C. Xin (dir.), Proceedings of World Conference on E-Learning in Corporate, Government, Healthcare, and Higher Education 2009 (p. 852-859). Association for the Advancement of Computing in Education (AACE).

Černochová, M., Voňková, H., Štípek, J. et Černá, P. (2018). How Do Learners Perceive and Evaluate Their Digital Skills?. International Journal of Smart Education and Urban Society, 9(1), 37-47. https://www.learntechlib.org/p/186429/.

Chiasson, M. (2019). Étude des caractéristiques de l'espace d'apprentissage favorisant le processus de la pensée informatique 


\section{8}

\section{REVUE HYBRIDE DE L'ÉDUCATION}

chez les élèves de l'école intermédiaire [thèse de doctorat inédite, Université de Moncton].

Cristol D. et Muller A. (2013). Les apprentissages informels dans la formation pour adultes. Savoirs, 32(2), 11-59. https://www.cairn.info/revue-savoirs-2013-2-page-11.htm

Conseil de recherche en sciences humaines (CRSH) (2010). Économie numérique: Acquérir les compétences numériques de demain. https://www.sshrc-crsh.gc.ca/home-accueil-fra.aspx

Donohoe, H. M. et Needham, R. D. (2009). Moving best practice forward: Delphi characteristics, advantages, potential problems, and solutions. International Journal of Tourism Reseach, 11(5), 415-437. https://doi.org/10.1002/jtr.709

Eagly, A. H. et Chaiken, S. (1993). The psychology of attitudes. Harcourt Brace Jovanovicha.

Ehlers, U.-D. et Kellermann, S. A. (2019). Future Skills - The Future of Learning and Higher education. Results of the International Future Skills Delphi Survey. Karlsruhe

Freiman, V., Berthoud, F., Cartier, J. et Chukalovsky, R. (2016). Un référentiel des compétences pour une société numérique: une perspective comparée des finalités technopédagogiques [communication orale]. $4^{\mathrm{e}}$ sommet de l'iPad et du numérique en éducation, Montréal, Qc, Canada.

Freiman, V., Godin, J., Chukalovskyy, R., Pelletier, W. et Paquet, M. (2015). Fifteen years of pedagogical advances of technology implementation in Atlantic Canada: from APTICA to CompéTICA. Dans S. Carliner, C. Fulford et N. Ostashewski (dir.), Proceedings of EdMedia : World Conference on Educational Media and Technology 2015 (p. 53-58). Association for the Advancement of Computing in Education (AACE).

Freiman, V., Godin, J., Larose, F., Léger, M. T., Chiasson, M., Volkanova, V. et Goulet, M. J. (2017). Towards the life-long continuum of digital competences: exploring combination of soft skills and digital skills development (p. 9518-9527). INTED2017 Proceedings, IATED.

Freiman, V., LeBlanc, M. et Léger, M. T. (2018). Citoyenneté numérique. Éduquer les jeunes Canadiennes et Canadiens à la citoyenneté numérique dans un contexte global: enjeux, défis, tendance, pratiques (Rapport final). Social Sciences and Humanities Research Council.

Furedi, F. (2018). How fear works: Culture of fear in the 21st Century. Bloomsbury Publishing.

Gapski, H. (2007). Some Reflections on Digital Literacy. Proceedings of the 3rd International Workshop on Digital Literacy (p. 49-55). 


\section{8}

\section{REVUE HYBRIDE DE L'ÉDUCATION}

Proceedings of the 3rd International workshop on Digital Literacy, CEUR-WS.org. http://ceur-ws.org/Vol-310/paper05.pdf

Godin, J., Léger M. T., LeBlanc M. et Freiman, V. (2020). Moving forward: Lessons learned from a collaboration network aimed at developing digital skills in New Brunswick. Proceedings Conference American Educational Research Association.

Gouvernement du Nouveau-Brunswick. (2003). Programme d'études : Sciences humaines - $5 e$ année. Ministère de l'éducation du Nouveau-Brunswick (MENB).

Gouvernement du Nouveau-Brunswick. (2006). Profil de sortie d'un élève du système scolaire acadien et francophone du Nouveau-Brunswick. Ministère de l'éducation et du développement de la petite enfance (MEDPE).

Gouvernement du Nouveau-Brunswick. (2018). Programme d'études. Mathématiques 30131 (9e année). Direction des programmes d'études. Ministère de l'éducation et du développement de la petite enfance (MEDPE).

Gouvernement du Québec. (2019). Continuum de développement de la compétence numérique - Cadre de référence de la compétence numérique. Ministère de l'Éducation et de l'Enseignement supérieur (MEES). http://education.gouv.qc.ca

Greene, K. (2018). Transferable Digital Literacy Knowledge. Language and Literacy Spectrum, 28(1). https://www.learntechlib.org/p/190951/

Hamutoglu, N. B., Savasci, M. et Sezen-Gultekin, G. (2019). Digital Literacy Skills and Attitudes towards E-learning. Journal of Education and Future, (16), 93-107. https://doi.org/10.30786/jef.509293

Inns, K., Nunn, J. et Surendranath, S. (2012). Building Research Partnerships - Developing a collaborative approach to support patient and public involvement (PPI) through learning and development. NCIR Cancer Conference Abstract.

Jenkins, H. (2007). Confronting the challenges of participatory culture: Media education for the 21 st century (Part One). Nordic Journal of Digital Literacy, 2(01), 23-33. https://www.idunn.no/dk/2007/01/confronting the challenges of $\mathrm{p}$ articipatory culture media education for the

Jones, S. H. et Putney, L.G. (2019). "Just the Beginning of Something Great:" A Telling Case of Action-Based Hope and Collective Classroom Efficacy. Journal of Ethnographic \& Qualitative Research, 13, 234-247.

LeBlanc, M., Léger, M. T., Godin, J., Freiman, V., Robichaud, X., Larose, F., Chukalovskyy, R. et Bourgeois, Y. (2015). A Strategic Partnership to Understand the Ecosystem, Adaptability and Transfer of Digital 


\section{$\&$}

\section{REVUE HYBRIDE DE L'ÉDUCATION}

Skills - a Focus on the Educational System. Proceedings of Society for Information Technology \& Teacher Education International Conference (p. 968-973). Advancement of Computing in Education (AACE).

Léger, M. et Freiman, V. (2016) A Narrative Approach to Understanding the Development and Retention of Digital Skills Over Time in Former Middle School Students, a Decade After Having Used One-to-One Laptop Computers. Journal of Research on Technology in Education. 48(1), 57-66.

Lum, L. et Tambyah, P. A. (2020). Outbreak of COVID-19 - an urgent need for good science to silence our fears?. Singapore Medical Journal, 61(2), 55-57. https://doi.org/10.11622/smedj.2020018

Martin, A. (2005). DigEuLit-a European framework for digital literacy: A progress report. Journal of eLiteracy, 2(2), 130-136. http://citeseerx.ist.psu.edu/viewdoc/download?

Martinovic, D. et Freiman, V. (2013). Digital skills development for future needs of the Canadian labour market (Rapport final). Social Sciences and Humanities Research Council.

McKinsey Global Institute. (2019). The future of work in America: People and places, today and tomorrow. McKinsey. https://www.mckinsey.com/featured-insights/future-of-work/thefuture-of-work-in-america-people-and-places-today-and-tomorrow

Miles, M. B. et Huberman, A. M. (1994). Qualitative data analysis : An expanded sourcebook ( $2^{\mathrm{e}}$ éd.). Sage Publications.

Meyers, E. M., Erickson, I. et Small, R. V. (2013). Digital Literacy and Informal Learning Environments an Introduction. Learning, Media and Technology, 38, 355-367.

Oberlander, M., Beinicke, A. et Bipp, T. (2020). Digital competencies: A review of the literature and applications in the workplace. Computers \& Education, 146, 1-13.

Organisation de coopération et de développement économiques (OCDE). (2013). Perspectives de l'OCDE sur les compétences 2013. Premiers résultats de l'Évaluation des compétences des adultes. http://dx.doi.org/10.1787/9789264204256-en

Organisation de coopération et de développement économiques (OCDE). (2019). Perspectives de l'OCDE sur les compétences 2019: Prospérer dans un monde numérique. https://doi.org/10.1787/23c2870c-fr

Orunsolu, A. A.,Vincent, O. R., Adebayo, A. A. et Bamgboye, O. O. (2010). Collaborative Research: A Synergistic Approach to Socio-Economic Development. Informing Science \& IT Education Conference (InSITE). 


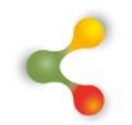

\section{REVUE HYBRIDE DE L'ÉDUCATION}

Paillé, P. (1994). L'analyse par théorisation ancrée. Cahier de recherche sociologique, 23, 147-181.

Phuapan, P., Viriyavejakul, C. et Pimdee, P. (2016). An Analysis of Digital Literacy Skills among Thai University Seniors. International Journal of Emerging Technologies in Learning, 11(3), 24-31. https://www.learntechlib.org/p/172230/

Powell, J. (2017). Defining and Assessing Digital Literacy. Dans P. Resta et S. Smith (dir.), Proceedings of Society for Information Technology \& Teacher Education International Conference (p. 1403-1406). Association for the Advancement of Computing in Education (AACE). https://www.learntechlib.org/primary/p/177909/

Raish, V. et Rimland, E. (2016). Employer Perceptions of Critical Information Literacy Skills and Digital Badges. College \& Research Libraries, 77(1), 87-113. https://www.learntechlib.org/p/194145/

Savard, A. et Freiman, V. (2012). Programmer un robot: quelles compétences mathématiques sont nécessaires. Colloque scientifique international sur les TIC en éducation : bilan, enjeux actuels et perspectives futures, Montréal, QC, Canada. http://ticeducation.org/files/actes/29.pdf

Siddiq, F., Gochyyev, P. et Wilson, M. (2017). Learning in Digital Networks - ICT literacy: A novel assessment of students' $21^{\text {st }}$ century skills. Computers \& Education, 109(1), 11-37. https://www.learntechlib.org/p/201637/

Statistique Canada. (2013). Les compétences au Canada: Premiers résultats du Programme pour l'évaluation internationale des compétences des adultes (PEICA). https://www.cmec.ca/Publications/Lists/ Publications/Attachments/315/Canadian-PIAAC-Report.FR.pdf

Tinder, K. Guiller, J. Margaryan, A., Littlejohn, A. et Nicol, D. (2008). Learning from digital natives: bridging formal and informal learning. The Higher education Academy.

Venkatesh, V. et Bala, H. (2008). Technology Acceptance Model 3 and a research agenda on interventions. Decision Sciences, 39(2), 273315.

Washbon, J. L. (2012). Learning and the new workplace: Impacts of technology change on postsecondary career and technical education. New Directions for Community Colleges, (157), 43-52.

Zorn, N. (2020). Pour une sortie de crise réussie : prioriser le $40 \%$ le plus défavorisé. Observatoire québécois des inégalités. 


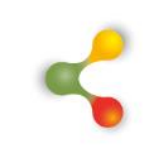

\section{REVUE HYBRIDE DE L’ÉDUCATION}

Remerciements : Cette étude a été réalisée grâce aux fonds de recherche obtenus du Conseil de recherche en sciences sociales et humaines (Développement de Partenariat \#890-2013-0062), la Fondation Innovation du Nouveau-Brunswick (2016, Programme Assistanat en recherche) et le Secrétariat aux Affaires Intergouvernementales Canadiennes du Québec (Programme de soutien à la Francophonie canadienne). 